\title{
Single-invariant nonlinear optics for a small electron recirculator
}

\author{
K. Ruisard, ${ }^{1, *}$ H. B. Komkov, ${ }^{2}$ B. Beaudoin, ${ }^{2}$ I. Haber, ${ }^{2}$ D. Matthew, ${ }^{2}$ and T. Koeth ${ }^{2}$ \\ ${ }^{1}$ Oak Ridge National Laboratory, Oak Ridge, Tennessee 37830, USA \\ ${ }^{2}$ Institute for Research in Electronics and Applied Physics, College Park, Maryland 20742, USA
}

(Received 16 November 2018; published 3 April 2019)

\begin{abstract}
This paper describes the design and simulation of a proof-of-concept octupole lattice at the University of Maryland Electron Ring (UMER). This experiment tests the feasibility of nonlinear integrable optics, a novel technique that is expected to mitigate resonant beam loss and enable low-loss high-intensity beam transport in rings. Integrable lattices with large amplitude-dependent tune spreads, created by nonlinear focusing elements, are proposed to damp beam response to resonant driving perturbations while maintaining large dynamic aperture. At UMER, a lattice with a single octupole insert is designed to test the predictions of this theory. The planned experiment employs a low-current high-emittance beam with low space charge tune shift $(\sim 0.005)$ to probe the dynamics of a lattice with large externally-induced tune spread. Design studies show that a lattice composed of a $25-\mathrm{cm}$ octupole insert and existing UMER optics can induce a tune spread of $\sim 0.13$. Stable transport is observed in PIC simulation for many turns at space charge tune spread 0.008. A maximum spread of $\Delta \nu=0.11$ ( $\mathrm{rms} 0.015$ ) is observed for modest octupole strength (peak $50 \mathrm{~T} / \mathrm{m}^{3}$ ). A simplified model of the system explores beam sensitivity to steering and focusing errors. Results suggest that control of orbit distortion to $<0.2 \mathrm{~mm}$ within the insert region is essential. However, we see only weak dependence on deviations of lattice phase advance $(\leq 0.1 \mathrm{rad}$.) from the invariant-conserving condition.
\end{abstract}

DOI: $10.1103 /$ PhysRevAccelBeams.22.041601

\section{INTRODUCTION}

One of several aspects limiting transportable beam current in accelerators is beam loss. Even low fractional losses in an intense beam present a hazard to personnel safety and the integrity of accelerator components as well as compromising beam quality. Known loss mechanisms include both incoherent and coherent resonances that drive particles to large amplitudes or lead to beam instability. This is especially of concern in high-intensity machines operating with large space charge tune spreads, which may encompass many periodic orbits of low order.

Moderate tune spreads have been known to stabilize beams against resonant interactions. In particular, nonlinear terms in the transverse focusing force can create a beneficial amplitude-dependent tune spread. The best known example is octupole-induced Landau damping, in which an octupole-induced tune shift in the particle distribution damps transverse collective instability [1]. In the presence of strong nonlinearity, regular driving terms cannot resonantly couple energy into the beam, as collective motions

\footnotetext{
*ruisardkj@ornl.gov
}

Published by the American Physical Society under the terms of the Creative Commons Attribution 4.0 International license. Further distribution of this work must maintain attribution to the author(s) and the published article's title, journal citation, and DOI. decohere and driven orbits shift away from the resonant condition [2]. The difficulty with imposing large tune spreads is the associated loss of dynamic aperture due to the emergence of chaotic orbits near resonance overlap. A solution has been proposed by Danilov and Nagaitsev in the theory of nonlinear integrable optics (NLIO) [3]. In this theory, integrable (nonchaotic) orbits are maintained for arbitrarily strong nonlinear focusing with large amplitudedependent tune shifts. Reference [3] identifies a family of nonlinear magnetic potentials in which transverse particle orbits conserve coupled, quadratic invariants of motion that are distinct from the Courant-Snyder invariants.

NLIO is a potential pathway for future high-intensity machine design, in which the traditional linear (quadrupole) optics are combined with strong nonlinear inserts. These nonlinear-focusing rings are predicted to be much more robust against single-particle resonances and coherent instability. Many numerical studies have been performed to gauge the feasibility of the NLIO concept, including the effect of lattice errors, dispersion, and chromaticity on integrability $[4,5]$. More recently, discussion has extended to understanding the contribution of space charge to invariants and beam stability [6]. A design study for a NLIO rapidcycling synchrotron has shown robust performance with space charge tune shifts $\Delta \nu=0.05$ [7]. However, at this point in time the concept is awaiting demonstration. Progress towards this goal is measured in the commissioning of 
the Integrable Optics Test Accelerator (IOTA), described in Ref. [8], as well as the effort described here.

An experimental program at the University of Maryland Electron Ring (UMER) has been developed as a testbed for the NLIO concept. The experiment is designed to produce a quartic Hénon-Heiles potential in the transverse confining forces. In this system, there is a single invariant of transverse motion. Compared to NLIO, the maximum stable tune spread induced on the particle distribution is expected to be smaller, but otherwise the lattice is similar in design. Unlike the regular orbits for fully integrable systems, some particle trajectories are expected to be chaotic yet still bounded. This program benefits from the flexibility of UMER to operate at a wide range of space-charge densities, which will provide insight into operation with space-charge beams at and beyond previous studies.

The design of a strongly nonlinear lattice is in itself a challenge, as there is no operational experience to draw on and the standard principles for accelerator design must be extended or modified. In the case of UMER, an additional constraint is found in seeking to implement an integrable optics design as an upgrade to an existing quadrupole lattice. We believe insights developed in retrofitting the UMER FODO lattice to accommodate octupole insertions, including a design for lightweight printed-circuit octupole insert and studies of predicted error sensitivity, may serve as a guide for future rings with integrable or single-invariant optics.

Section II reviews the basic parameters of the UMER lattice. Section III is an overview of the design of an octupole lattice for UMER. This section describes both development of the octupole insert as well as optimization of the linearfocusing optics for invariant-conserving transport and the available beam parameters for experiments. In Sec. IV, particle-in-cell (PIC) simulations are used to explore particle dynamics in the proposed lattice. Conclusions are drawn regarding optimal operating point and required tolerances. Finally, Sec. V provides discussion of the lessons learned and outlook for the UMER nonlinear optics program.

\section{UMER PROGRAM}

UMER is a scaled, $10 \mathrm{keV}(\beta=0.195)$ electron ring experiment for the study of high-intensity beam dynamics relevant to higher-energy ion rings. A range of space charge densities are selected by aperturing the beam near the source, in the range $\nu / \nu_{0}=0.95$ to 0.14 for nominal tune 6.7 (incoherent shifts $\Delta \nu=\nu_{0}-\nu=0.3$ to 5.7) $[9,10]$. Here $\nu$ is betatron tune for a particle at peak density and $\nu_{0}$ is bare tune. As such, typical operation in UMER exceeds the space charge intensity of existing, production-level rings such as $\nu / \nu_{0}=0.98, \Delta \nu=0.15$ in the SNS accumulator ring [11].

The built-in flexibility of UMER is easily leveraged for nonlinear optics experiments. The linear optics are highly configurable thanks to independently-powered quadrupoles. The low beam rigidity allows for the use of low-cost, air-core printed circuit board (PCB) magnets. The same approach is applied in design of the nonlinear insert for UMER. The lattice also includes induction cells for longitudinal focusing, which supports multiple confinement conditions such as rf sine (recently implemented) and rf barrier bucket in addition to unconfined/coasting bunches $[12,13]$.

In retrofitting any experiment for a new configuration, there are challenges. For UMER these included the high density of ring optics, large closed orbit distortion, and the large space-charge tune depression which exceeds the predicted octupole-induced spread. Each of these points is expanded on where appropriate below.

\section{DESIGN OF OCTUPOLE LATTICE}

The defining attribute of a NLIO lattice are nonlinear insertion elements embedded within a linear-focusing lattice. Both the shape of the nonlinear potential and the transfer function of the linear-focusing lattice must be tailored to ensure integrability. The recipe laid out in Ref. [3] is reiterated here for convenience. In this approach, constant Laplacian potentials are identified that permit one or two invariants of motion. The nonlinear potential is constant in normalized phase space coordinates ${ }^{1}$ and therefore has lab-frame longitudinal dependence that scales with the betatron function $\beta(s)$, where exact scaling depends on pole order in the multipole expansion of the nonlinear potential. For proper cancellation of terms, the beam must be round through the nonlinear insert, $\beta_{x}(s)=\beta_{y}(s)$. In implemention, linear-focusing ring sections are tailored to form a round waist at the insert location. Finally, for quasicontinuous motion through the nonlinear potential the phase advance in the linear-focusing optics between inserts must be integer $\times \pi$. This of course means that any additional contribution to particle trajectories, such as space charge depression or off-momentum effects, causes orbits to shift away from the known invariant [4].

\section{A. Nonlinear insert}

An early choice for the UMER lattice was to test an invariant-conserving, nonintegrable octupole lattice rather than the fully 2D-integrable case. This is motivated by the expectation of relaxed tolerances in the single-invariant system when compared to NLIO (which requires control of betatron tune to within $2 \pi \times 10^{-3}[8]$ ) and the significantly reduced complexity for design of a single-multipole magnetic potential. In particular, the UMER PCB magnets can easily be configured for any $\cos n \theta$ current distribution [14]. ${ }^{2}$ A similar system with octupole inserts is planned at the IOTA ring, where the desired tolerance for phase advance is $2 \pi \times 10^{-2}[16]$.

$$
{ }^{1} x_{N} \equiv \frac{x}{\sqrt{\beta_{x}(s)}}, p_{x, N} \equiv p_{x} \sqrt{\beta_{x}(s)}+\frac{\alpha_{x} x}{\sqrt{\beta_{x}(s)}} .
$$

${ }^{2}$ This is in contrast to the IOTA nonlinear inserts, in which iron pole-pieces form both an octupole and a fully integrable potential $[8,15]$. 

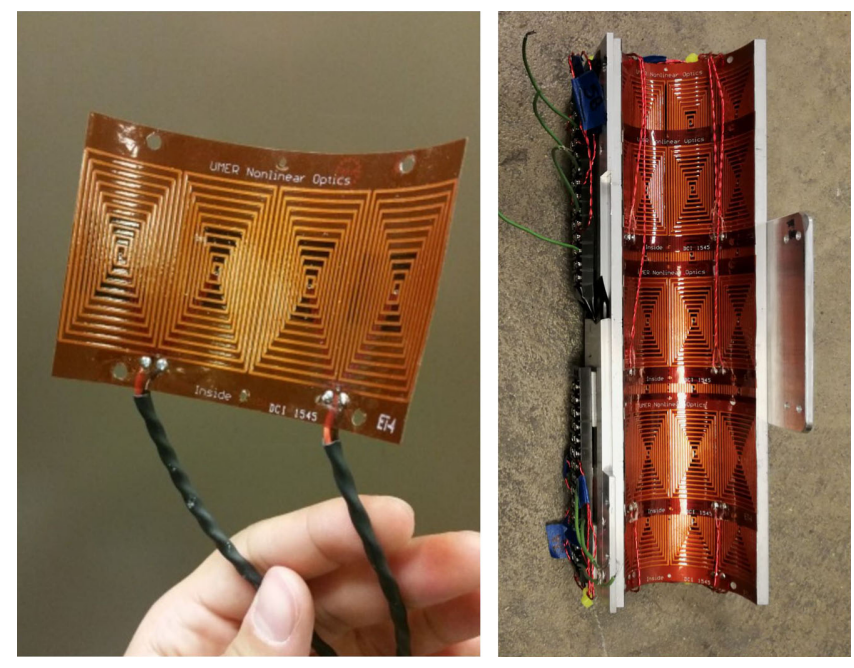

FIG. 1. Left: Half of printed circuit octupole magnet. Right: top half of $25 \mathrm{~cm}$ composite octupole channel containing seven $4.65 \mathrm{~cm}$ PCBs.

In the case of octupole fields, the single invariant of transverse motion takes the form (in normalized canonical coordinates) [3]

$H_{N}=\frac{1}{2}\left(p_{x, N}^{2}+p_{y, N}^{2}+x_{N}^{2}+y_{N}^{2}\right)+\frac{\kappa}{4}\left(x_{N}^{4}+y_{N}^{4}-6 y_{N}^{2} x_{N}^{2}\right)$.

Following from the $x^{4}$ dependence of the fields, the octupole strength parameter $\kappa$ is s-independent (and $H_{N}$ is invariant) if the octupole term has dependence proportional to $\beta^{-3}(s)$.

The Hamiltonian in Eq. (1) is a member of the well-studied Hénon-Heiles family of equations with nonlinear, coupled polynomial potentials [17]. In general these systems are nonintegrable, however there are regions for which orbits are regular. Motion is expected to remain bounded even in chaotic regions, due to the invariant amplitude $H_{N}$. The implications for stability when "chaotic but bounded" orbits are introduced are still unclear-see additional discussion in Sec. IVA.

The nominal UMER FODO lattice is very dense (magnet fill factor 43\%), due to the need to provide confinement for very high intensity beams. With 36 bends in an 11.52 meter circumference, the longest straight section available for an insertion device is $25 \mathrm{~cm}(32 \mathrm{~cm}$ minus mechanical clearances). To maximize configurability, short $4.65 \mathrm{~cm}-$ long octupoles were fabricated as flexible PCBs, shown in Fig. 1, and assembled into a composite "octupole channel" consisting of seven independently-powered octupole circuits $[18,19]$. The radially-thin PCBs overlap within the channel to produce a fairly smooth longitudinal profile $K_{3}(s)$ as shown in Fig. 2. There is $2 \%$ rms flutter about the ideal profile $\propto \beta(s)^{-3}$, but numerical studies predict minimal effect on dynamic aperture. Individual PCB octupoles have a peak gradient strength $51.6 \pm 1.5 \mathrm{~T} / \mathrm{m}^{3} / \mathrm{A}$ and unwanted

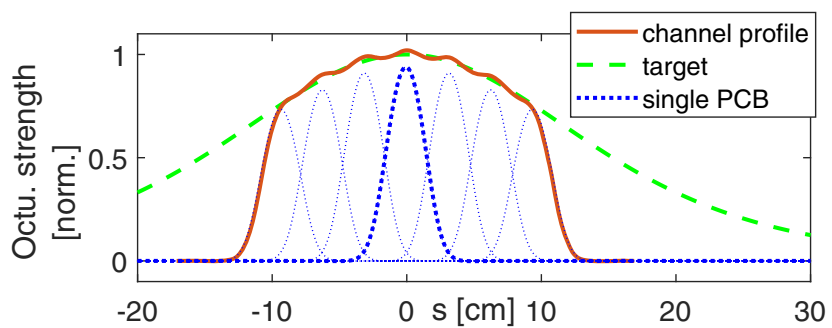

FIG. 2. Longitudinal profile of composite octupole channel with overlapping, evenly spaced PCBs. Profile is determined from Biot-Savart integration of the designed circuit.

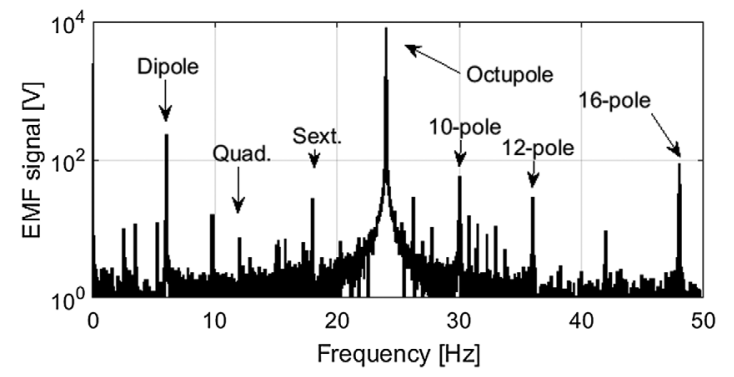

FIG. 3. FFT from rotating coil measurement of single (short) octupole magnet. Large dipole peak is due to presence of ambient Earth magnetic field and does not originate from octupole circuit.

multipole orders are suppressed by two orders of magnitude as measured by a rotating coil, shown in Fig. 3.

\section{B. Choice of operating point}

The fractional tunes of the lattice, as determined by the linear optics, should be equal to the total "tune advance" $\nu_{\mathrm{ch}}$ through nonlinear insertions (or equal to $\frac{1}{2}-\nu_{\text {ch }}$ ) to meet the integer $\times \pi$ requirement introduced above. This fractional tune can be chosen to maximize tune spread; it is observed in simulation that maximum induced tune spread is approximately equal to the fractional tune, that is $\Delta \nu_{x}=$ $\Delta \nu_{y} \sim \nu_{\mathrm{ch}}$. Therefore, largest possible tune advance is desired, which corresponds with small beam waist in the insert region (in this way the lattice design is similar to that of mini-beta insertions for colliders). Here we use notation $\beta_{*}$ to indicate the betatron function at the waist. To avoid scraping of a $100 \mu \mathrm{m}$ beam in the quadrupoles flanking the insert region, $\beta_{*}>0.23 \mathrm{~m}$ is required (using a $2 \times$ edge safety factor from the $5 \mathrm{~cm}$ pipe diameter). For the UMER design, $\beta_{*}=0.3 \mathrm{~m}$ is identified as a feasible target for largest possible phase advance that can be supported by existing optics. For a single $25-\mathrm{cm}$ octupole insert, the ring operating point should be $\nu_{x}=\nu_{y}=0.13$. and the limiting, maximum tune spread (across all stable orbits) is $\sigma_{\nu}=0.13$, This is verified by simulations in a simple, reduced model described in Sec. III E.

The octupole strength parameter $\kappa$ should be chosen to maximize amplitude-dependent tune spread but also ensure sufficient dynamic aperture to avoid particle losses. In the 


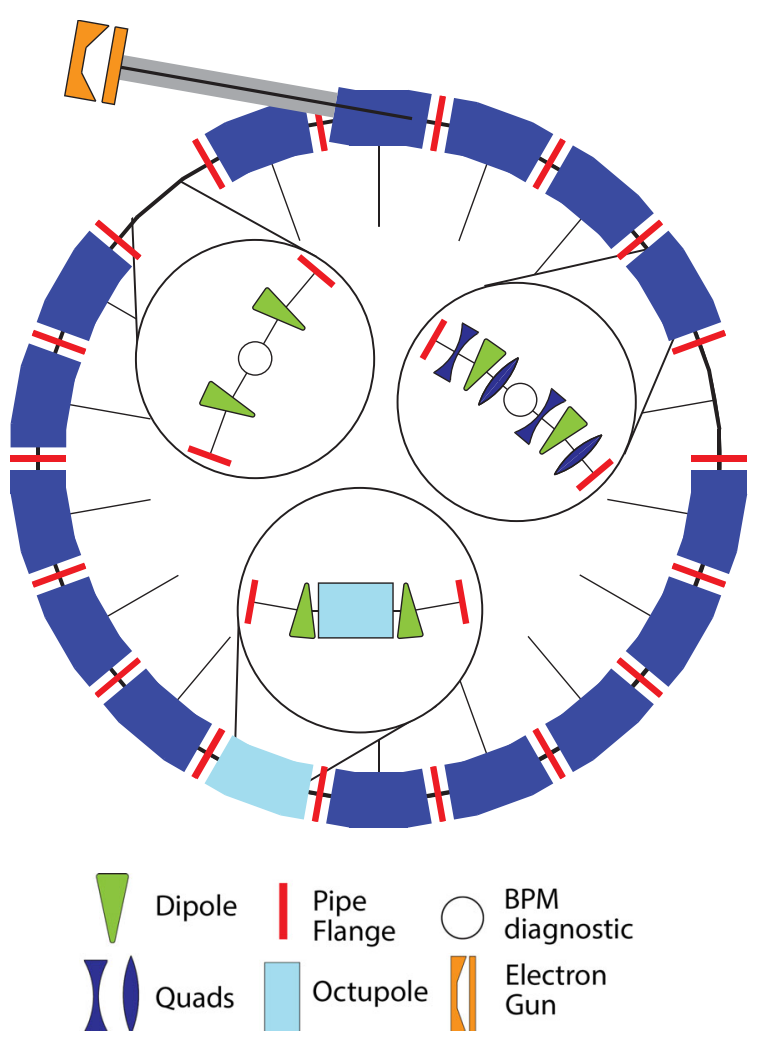

FIG. 4. Diagram of UMER octupole lattice, with insets showing detail for standard $20^{\circ}$ section (Q-D-Q-Q-D-Q) and octupole section (0-D-OCTU-D-0). Spokes indicate diagnostic locations and red bands indicate vacuum flanges.

octupole lattice, there is a sharp boundary to the dynamic aperture and the induced tune shift is largest for particles near the aperture limit. This limit shrinks with increased octupole strength and is bounded by unstable fixed points at $\left(x_{N}, y_{N}\right)=( \pm 1 / \sqrt{2 \kappa}, \pm 1 / \sqrt{2 \kappa})$, first described in Ref. [20]. Therefore, the radial limit for stability appears at $r_{\max }=\sqrt{\beta_{*} / \kappa}$. Due to the shape of the separatrix and unbounded orbits near the fixed point, this represents an upper bound. Assuming an initially round beam, the effective stable aperture is typically closer to $70 \%$ of this value.

For the $25-\mathrm{cm}$ insert over a $\beta_{*}=0.3 \mathrm{~m}$ waist, a peak octupole gradient of $50 \mathrm{~T} / \mathrm{m}^{3}$ places the fixed point at $3.2 \times \mathrm{rms}$ radius for a $100 \mathrm{~mm}$-mrad edge-emittance beam. This requires only $0.97 \mathrm{~A}$ in the central octupole circuit, well within the safety limit of the UMER PCB octupoles. It is worth noting that for the UMER experiments, we aim to operate near the aperture limit (filling the ring acceptance) for maximum nonlinear effect. In a system requiring large dynamic aperture, the expected tune spread will be significantly reduced from the above estimate $\left(\delta \nu \sim \nu_{\text {frac }}\right)$ as the largest spreads are found very near the stability boundary.

\section{Linear-focusing optics}

The layout of the UMER nonlinear optics experiment is shown in Fig. 4, while Table I summarizes the parameters
TABLE I. Nominal parameters for the UMER octupole lattice.

\begin{tabular}{lc}
\hline \hline Parameter & Value \\
\hline Beam energy & $10 \mathrm{keV}$ \\
Beam current & $0.04-0.6 \mathrm{~mA}$ \\
Incoh. $\Delta \nu$ & $0.005-0.94$ \\
Circumference & $11.52 \mathrm{~m}$ \\
Pipe diameter & $5.08 \mathrm{~cm}$ \\
Bunch length & $20-100 \mathrm{~ns}$ \\
Super-period & $3.84 \mathrm{~m}$ \\
Quadrupoles per period & 20 \\
Bare tune $\left(\nu_{x}=\nu_{y}\right)$ & $3.13(3.1-3.4 \mathrm{range})$ \\
Natural chromaticity & $C_{x}=-4.3$ \\
& $C_{y}=-3.2$ \\
\hline \hline
\end{tabular}

for the lattice as designed. The design includes a single nonlinear insertion for proof-of-principle experiments. In general, multiple insertions should lead to stronger resonance damping and room is held in this design for additional octupole inserts with minimal modification. As the ring is built in modular $20^{\circ}$ arcs, each housing two dipole bends and four quadrupoles (Q-D-Q-Q-D-Q), an entire $20^{\circ}$ section is modified to accommodate the insertion. The long insert occupies the straight between dipoles (0-D-OCTU-D-0), as shown via inset in Fig. 4.

The only fixed parameters in the linear lattice design are the magnet locations. There are two quadrupoles per dipole (72 total) at equal $16 \mathrm{~cm}$ spacing. To make room for a long insert it is necessary to physically remove two quadrupole elements, leaving 70 available for meeting the integer $\times \pi$ requirement. A few-period solution is chosen, shown in Fig. 5, in which 60 of the available quadrupoles are used for the linear focusing in periodic arrangement of 10 unique strengths (optimization problem is reduced to 10 degrees of freedom).

The choice of a few-period design with many quadrupoles per period allows for flexibility of lattice function (i.e., shifting operating points in linear lattice without changing optics of insert region) and supports largely adiabatic changes to the beam size (empirically observed to reduce

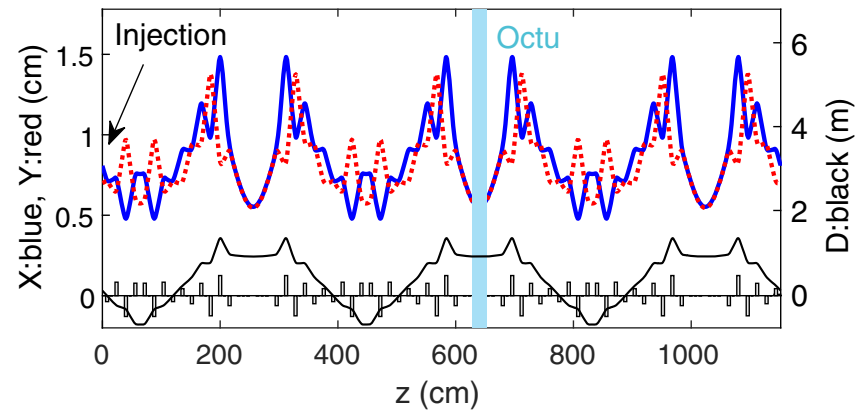

FIG. 5. Periodic KV envelope solution for $100 \mathrm{~mm}-\mathrm{mrad}$, $60 \mu \mathrm{A}$ beam at $\nu_{x}=3.124, \nu_{y}=3.128$. Injection is at $s=0$ plane. 
sensitivity to quadrupole strength errors). In this case, superperiodicity of six is theoretically possible ( 8 quads per cell, 4 degrees of freedom) but this restricts flexibility in selecting tune. ${ }^{3}$ Periodicity four is more tractable, but periodicity three is chosen to align with diagnostics (located at periodicity eighteen).

The design shown in Fig. 5 assumes a low-current, large-emittance beam $(40 \mu \mathrm{A}, 100 \mathrm{~mm}-\mathrm{mrad} 4 \times \mathrm{rms}$, unnorm.) for low space charge tune spread during initial experiments. The tune of the lattice shown is $\nu_{x}=3.124$, $\nu_{y}=3.128$. This is much lower than the typical operating parameters for $\operatorname{UMER}\left(\nu_{x}=6.68, \nu_{y}=6.69\right)$. The weaker focusing allows for long $(64 \mathrm{~cm})$ quadrupole-free sections while avoiding unacceptably large excursions of the beam envelope.

There is some flexibility in choosing the orientation of the three-period lattice solution with respect to injection. Six configurations are possible, as each $120^{\circ}$ superperiod is comprised of six $20^{\circ}$ modular sections (Q-D-Q-Q-D-Q). The orientiation shown in Fig. 4 is chosen based on two primary considerations: low local closed orbit distortions at the insert location (as measured in typical operation) and injection where the horizontal beam size is small. ${ }^{4}$

\section{Off-momentum orbits}

Maintaining momentum acceptance is important for nonlinear ring design. In general, off-momentum orbits will not meet the conditions for conserved invariants. The effects on a NLIO lattice are discussed in Ref. [4], which recommends that chromaticities be equal and dispersion as low as possible. While the UMER lattice includes neither chromaticity-correcting sextupoles nor a dispersion-matching section at injection, low momentumdeviation is generally expected in coasting beam and rf-confined experiments. Unconfined beam from the gridded triode electron source is expected to generally have low energy spread, $\delta p / p \sim 0.001$, except in the eroding head and tail of the bunch when confining fields are off $[21,22]$. Energy spread is not expected to increase significantly with rf confinement, as low-charge beams are seen in operation to require very low $\mathrm{rf}$ voltages [12].

With natural chromaticities $C_{x}=-4.3, C_{y}=-3.3$, the expected coasting beam chromatic tune spreads are $\sim 0.004$. If necessary, this may be corrected by the addition of PCB sextupole elements. For the matched dispersion in Fig. 5 we expect $x_{D}=0.09 \mathrm{~cm}$ in the octupole for a $+10 \mathrm{eV}$ error in energy. This leads to additional terms in the invariant $H_{N}$

\footnotetext{
${ }^{3}$ Periodicity refers to the nominal linear optics design. Due to modified optics at injection, the true superperiodicity of the UMER is one.

${ }^{4}$ Due to a peculiarity in the UMER injection design, injection cannot be done in one of the long drifts as an off-axis quadrupole is used for simultaneous focusing and bending of the beam into the ring.
}

[Eq. (1)] that may lead to diffusion. The case for unmatched dispersion has yet to be addressed, but the dependence can be probed in experiment as well as in simulation.

\section{Electron beam parameters for nonlinear optics experiments}

As the invariants found in Ref. [3] are calculated in the single particle limit, initial tests of the octupole lattice will focus on beams with weak space charge prior to exploring higher intensity regimes. The parameters for all available UMER beams are given in Table II. The "standard" UMER beams, generated using a single aperture at the source, occupy rows three and below $(\geq 0.6 \mathrm{~mA})$. Clearly, the incoherent tune shifts $\geq 0.94$ far exceed the predicted octupole-generated tune spread $(\leq 0.13)$. The beams described in rows one and two were developed in preparation for the nonlinear optics experiments.

The $60 \mu \mathrm{A}$ beam (second row in Table II) is generated using an aperture-solenoid-aperture system to reduce beam density while maintaining low emittance, and is described in Refs. [10,23]. The nominally $40 \mu \mathrm{A}$ beam (first row in Table II) is generated by operating the UMER triode electron gun in voltage amplification mode [24]. In this mode the output current depends strongly on the bias voltage in the triode, allowing tuning of emitted current in the range 0.01$0.1 \mathrm{~mA}$. This beam has low incoherent tune shift due to the large emittance; quadrupole scan slice-emittance measurement at $40 \mu \mathrm{A}$ output current yields $\epsilon_{x}=300 \mathrm{~mm}$-mrad, $\epsilon_{y}=100 \mathrm{~mm}$-mrad (unnorm., $4 \times \mathrm{rms}$ ) at the midpulse location. The large asymmetry in this measurement is likely due to the time-varying injection kick or large mismatch as profile measurement near the source indicate that $\epsilon_{x} \approx \epsilon_{y}$.

\section{E. Simulation tools}

The performance of the octupole lattice depends on correct execution of the linear-focusing lattice as well as the precision of the octupole insert. The effects of lattice errors

TABLE II. UMER beam parameters. The top three rows are proposed for nonlinear optics experiments. $r_{0}$ is radius of aperture used to form beam, emittance $\epsilon$ is unnormalized rms emittance, $\nu / \nu_{0}$ values are estimated for FODO lattice with operating point $\nu_{x}=\nu_{y}=6.8$, and tune shifts are estimated for a $\mathrm{KV}$ equivalent beam.

\begin{tabular}{lcccc}
\hline \hline Current $[\mathrm{mA}]$ & $r_{0}[\mathrm{~mm}]$ & $\epsilon[\mathrm{mm}-\mathrm{mrad}]$ & $\nu / \nu_{0}$ & Incoh. $\Delta \nu$ \\
\hline $0.04(0.01-0.1)$ & $\ldots$ & $25 \pm 5$ & 1.00 & -0.005 \\
$0.06^{\mathrm{a}}$ & 0.7 & 2.5 & 0.95 & -0.3 \\
0.6 & 0.25 & 7.6 & 0.85 & -0.94 \\
6.0 & 0.875 & 25.5 & 0.62 & -2.4 \\
21 & 1.5 & 30.0 & 0.31 & -4.5 \\
80 & 2.85 & 86.6 & 0.17 & -5.5 \\
100 & 3.2 & 97.3 & 0.14 & -5.7 \\
\hline \hline
\end{tabular}

${ }^{\mathrm{a}}$ See Ref. [10] and [23]. 
are examined in simulation. PIC simulations are performed using the transverse-slice package in the WARP code [25], in which the beam is assumed to be uniform and infinite along the longitudinal axis. We refer to two models of the octupole lattice: a "simple model" of the nonlinear lattice, consisting only of the octupole element and an ideal thinlens symmetric-focusing kick as a proxy for the linear focusing section, and a full ring model, which uses hardedged models for the quadrupoles. in the full ring model used here, dipoles are excluded for simplicity and ease of tuning. The UMER dipoles introduce a vertical focusing component due to the fringe fields and a sextupole term introduced by the PCB windings. Lattice solutions including the dipole contributions to linear focusing have been found and appear very similar to the solution shown in Fig. 5 (see for example Ref. [26]) but are not reported on here. For the octupole insert, we implement both an ideal octupole potential with smoothly varying longitudinal profile or gridded field model representative of the octupole channel as designed. The gridded model is generated by integrating the Biot-Savart law for the PCB current distribution using in-house code MAGLi [27].

In the PIC simulation, we sample the frequency map [28] with a zero-charge, gridded "witness distribution". 40k macro-particles are used to calculate the space charge force every timestep, which is set for one millimeter of forward motion per step. A convergence study was performed using the simple model at peak octupole strength $50 \mathrm{~T} / \mathrm{m}^{3}$. For 40k particles the error in rms beam moments after 1024 turns was within $0.5 \%$ of the limiting values. The test case beam is initialized as a $60 \mu \mathrm{A}$ semi-Gaussian distribution with $100 \mu \mathrm{m}$ edge emittance in both planes. In simulation this is measured to have an incoherent tune spread of $\Delta \nu=-0.008$. This is a slightly higher current than the nominal case for the low-current beam (Table II, first row).

\section{DYNAMICS IN OCTUPOLE LATTICE}

\section{A. Chaotic motion}

The potential described in Eq. (1) is a quartic case of the generally nonintegrable Hénon-Heiles dynamical system. As is characteristic of these systems, the octupole potential described here preserves regular, nonchaotic orbits for some initial conditions, while exhibiting chaotic (but bounded) trajectories at others [17]. Figure 6 displays characteristics of the ideal map in Eq. (1) for trajectories with initial conditions $\left(x, y^{\prime}\right)=(0,0)$. Some features of note are that the boundary of stability (black contour) covers a much wider area than the boundary that roughly outlines the location of regular orbits (magenta contour). This boundary indicates where a small error in initial condition grows by less than a factor of 10 after 100 oscillations. The inset plots in Fig. 6 show surface-of-section maps for a set of particles launched along an equipotential curve in $\mathrm{X}$ and $\mathrm{Y}$, showing the emergence of stochastic motion at higher amplitudes.

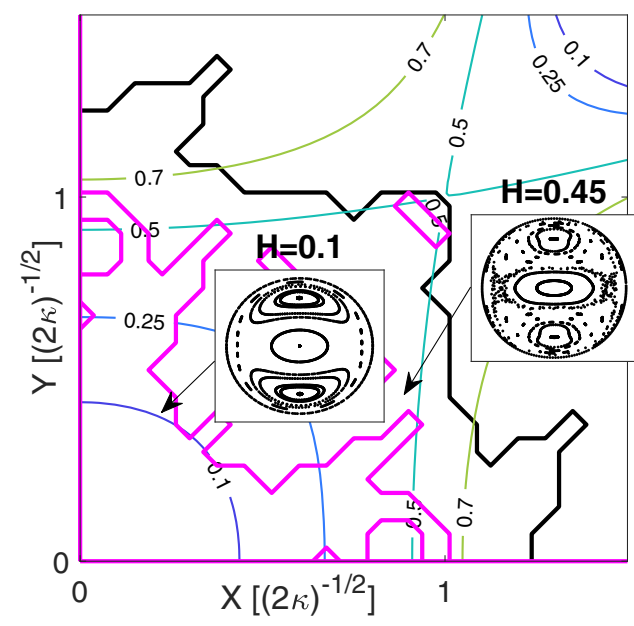

FIG. 6. Regions of stability (black curve) and regularity (magenta curve) for particles with initial conditions $\left(x, y^{\prime}\right)=$ $(0,0)$. Equipotential lines are shown for $\kappa=0.5$, for which the separatrix occurs at $H_{N}=0.5$. The inset plots show the $x=0$ surface-of-section for $\mathrm{y}-\mathrm{y}$ motion at fixed $H_{N}$.

This "chaotic-but-bounded" lattice is significantly different from the Courant-Snyder system, where all orbits in the ideal map are regular. The boundedness is ensured by invariant conservation, which limits particle amplitude. Within the separatrix, particle motion is expected to be confined. Regions of apparent stability appear beyond the separatrix along the $\mathrm{X}$ and $\mathrm{Y}$ axes, but orbits in these wings are not bounded. The strength of the UMER octupole insert is sufficiently strong to place the separatrix near the edge of the bunch for the $0.04 \mu \mathrm{A}$ beam (Table II, first row) and the test case $0.06 \mu \mathrm{A}$ used in simulations below.

\section{B. Transport at design operating point}

The nominal lattice design, as described above, is for a single-insert lattice with $25-\mathrm{cm}$ octupole insert over $\beta_{*}=$ 0.3 waist, with linear optics optimized to be near the invariant-conserving condition $\nu_{x}=\nu_{y}=0.126$. Figure 7 shows the result of PIC calculations at peak octupole strength $50 \mathrm{~T} / \mathrm{m}^{3}$, including the sampled frequency map and output beam distribution. The dynamic aperture limit can clearly be seen in the frequency map shown in Fig. 7(b), as well as the characteristic eight-lobed structure imprinted by the octupole potential. There is excellent agreement with the equivalent simple model $(25-\mathrm{cm}$ channel + thin-lens focusing kick), whose stability boundary is shown as the light green contour in Fig. 7(b). The "airplane" shape of the tune footprint in Fig. 7(a) is also characteristic, with highamplitude particles being shifted to the tips of the "wings". Particle color in these plots represents a frequency shift $d \nu$ over the length of the simulation, where higher values correspond with more irregular motion indicating a chaotic trajectory or proximity to resonance.

The slight tilt in both tune and configuration space is characteristic of a small tune asymmetry in the PIC model, 


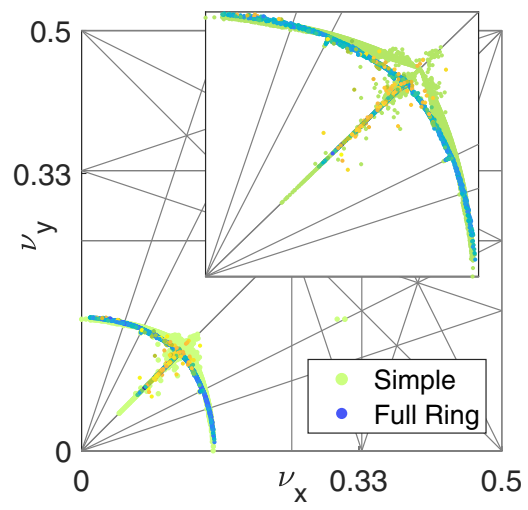

(a) Tune footprint with up to fourth order resonance lines indicated. For color axis refer to scale in plot below.

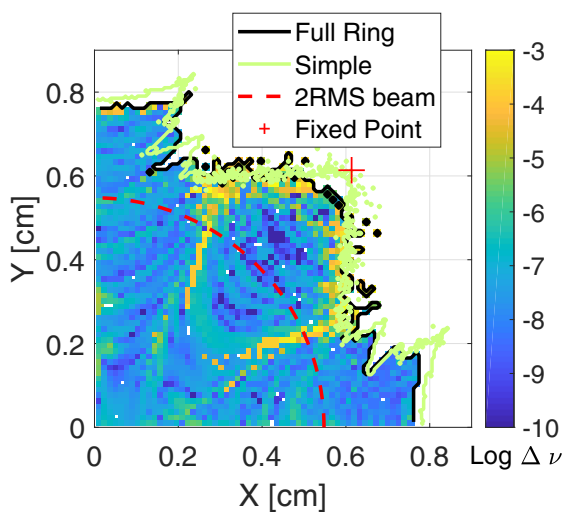

(b) Configuration space plot of aperture and resonant structure as a function of particle initial condition. The fixed point at $x=y=\sqrt{\beta_{*} / 2 \kappa}$ is indicated here by the red cross marker.

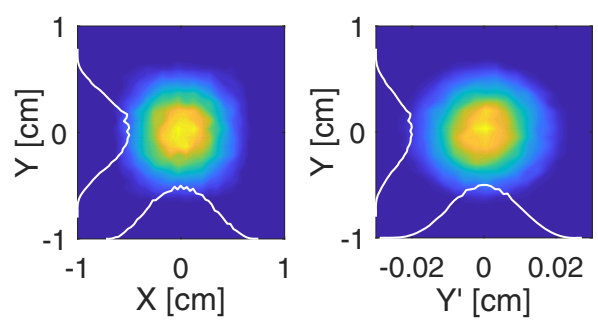

(c) Snapshot of beam distribution at beam waist. Color axis scales linearly with beam density.

FIG. 7. WARP PIC calculation for octupole lattice with peak strength $50 \mathrm{~T} / \mathrm{m}^{3}\left(\kappa=3984 \mathrm{~m}^{-1}\right)$ and fractional tune 0.13 after 1024 turns.

with $\nu_{x}-\nu_{y}=-0.001$. The simulated tune spreads are $\Delta \nu_{x}=-0.10, \Delta \nu_{y}=-0.11$ and at the given peak strength $50 \mathrm{~T} / \mathrm{m}^{3}$, dynamically unstable orbits appear at $2.27 \times \mathrm{rms}$ radius. The effect of tune depression at the bunch center can be seen in the tune diagram [Fig. 7(a)]. A snapshot of the bunch distribution in Fig. 7(c) shows a slight "squaring" in configuration space, which is expected for the octupole potential.
Transport at this operating point is summarized in the first row of Table III. Conservation of the invariant $H_{N}$ for stable orbits (still confined at 1024 turns) is shown in the last column. In general, orbits exhibit small oscillations $<1 \%$ in the zero-charge limit. Including the space charge contribution causes larger oscillations, although orbits still appear to be bounded. Within the first 30 turns, there is a rapid redistribution of charge which is reflected in sharp changes in single particle amplitudes $H_{N}$. The contribution of the initial redistribution to the quantity $\operatorname{std}\left(H_{N}\right) /\left\langle H_{N}\right\rangle_{t}$ in Table III is small $(+0.1 \%)$. The dominant contribution is the invariant-breaking effect of space charge at low amplitudes, which causes relatively large $H_{N}$ variation for orbits with small $\left\langle H_{N}\right\rangle_{t}$.

It is worth pointing out that for the octupole lattice, beam emittance is no longer explicitly conserved, as is the case for linear lattices in which the emittance is equivalent to the Courant-Snyder invariant. In the octupole lattice, the initial emittance of the injected beam is not conserved, as nonlinear forces cause filamentation of the phase space volume and any initial beam mismatch is quickly converted to growth of the emittance term (first detailed in Ref. [4]). However, this growth stabilizes quickly as the filamentation is rapid in a strongly nonlinear system. Therefore, tracking rms emittance is still a useful tool, as it does appear to reach an equilibrium value and can easily be related to beam size in linear sections of transport.

\section{Operation near a fourth-order resonance}

One aim of the UMER octupole lattice design was to maximize the induced tune spread, which scales with the fractional tune. An earlier version of the design assumed a $64 \mathrm{~cm}$ octupole section, in which the central $25 \mathrm{~cm}$ insert is flanked on either side by one to two short octupole PCBs. This creates a longer octupole channel but with discontinuities to accommodate the two $10^{\circ}$ bends. This was shown in simulation to lead to large loss of stable orbits and this scheme was abandoned. (For more details, refer to Ref. [24] and [29].) PIC simulations are still run for this configuration, using just the $25 \mathrm{~cm}$ insert but with tune $\nu_{x}=\nu_{y}=$ 3.263 optimized for a $64 \mathrm{~cm}$ insert over $\beta_{*}=0.3 \mathrm{~m}$ waist.

Results are shown in Fig. 8. There is very poor agreement with the predictions of an equivalent simple model, including severe loss of previously stable areas that reduces the spread of tune. While the lattice tune is far from the invariant-conserving condition, the greater effect is the nearness of the lattice tune to the fourth order $\nu_{x}, \nu_{y}=0.25$ resonances, which are strongly driven by the octupole term. Figure 8(c) depicts clear fourth-order resonant structure in the beam distribution. Loss of orbit stability appears to coincide with the overlap of the fourth order resonance with nearby sum resonances $3 \nu_{x}+\nu_{y}=1, \nu_{x}+3 \nu_{y}=1$ and $2 \nu_{x}+2 \nu_{y}=1$.

Studies at other operating points showed similar losses near $\nu_{x}=0.25$. In one case with fractional tune $\nu_{x}=0.24$, 
TABLE III. Predicted performance of two tune operating points for PIC simulation of octupole lattice with hardedged elements and peak octupole gradient $50 \mathrm{~T} / \mathrm{m}^{3}\left(\kappa=3984 \mathrm{~m}^{-1}\right)$. Tune spreads are calculated as difference from bare tune in linear lattice; the maximum spread is calculated for all stable orbits, while the rms spread is calculated for all orbits within $2 \times r m s$ of the bunch center. Conservation of invariant $\operatorname{std}\left(H_{N}\right) /\left\langle H_{N}\right\rangle_{t}$ is taken as average over all stable particle orbits within $2 \times r m s$ beam radius.

\begin{tabular}{lcccc}
\hline \hline Tune & $\Delta \nu_{x} \max / r m s$ & $\Delta \nu_{y} \max / r m s$ & eff. $r_{\max }$ & $\left\langle\operatorname{std}\left(H_{N}\right) /\left\langle H_{N}\right\rangle_{t}\right\rangle 0 \mathrm{~A} / 60 \mu \mathrm{A}$ \\
\hline 0.13 & $0.101 / 0.015$ & $0.110 / 0.016$ & $0.62 \mathrm{~cm}$ & $0.91 \% / 6.54 \%$ \\
0.26 & $0.042 / 0.007$ & $0.050 / 0.005$ & $0.40 \mathrm{~cm}$ & $8.02 \% / 24 \%$ \\
\hline \hline
\end{tabular}

the resonant losses resulted in hollowing of the beam core without disrupting stable orbits at higher amplitudes. Limiting the tune below $\nu=0.25$ avoids these losses, at the detriment of reducing the octupole-induced tune spread. Due to the high-emittance test beam and fixed pipe aperture, it is not possible to operate sufficiently far above $\nu=0.25$ to avoid this resonance. Thus, the fourth order resonance acts as an upper bound for operating points for single-insert experiments at UMER.

It is clear that the stability loss is a function of proximity to the fourth-order stop-band. As this behavior emerged only after addition of the full lattice structure, it is likely that parametric resonance driven by the lattice contributes to the loss. However, stability of simulations without the octupole insert indicates strong dependence on the insert as a driver. In general, for octupole lattice design it may be necessary to avoid spanning the fourth order stop-bands. It is worth pointing out this case is far from the invariantconserving condition (with error in betatron tune $\delta \nu \sim 0.13$ ) when compared to the simple model (green trace in Fig. 8) which exactly meets the condition. An invariant-conserving lattice with similar tune operating point may demonstrate greater stability despite the nearby resonance.

\section{Tolerances for closed-orbit distortion and particle phase errors}

A primary concern for the UMER nonlinear optics experiments is the error sensitivity of the octupole lattice relative to UMER tolerances as they are known. The two biggest areas of concern are transverse misalignment and errors in the linear focusing function. The simple model described above (octupole insert and thin-lens focusing kick) was used to interrogate the effect of beam misalignment and incorrect linear optics. This approach is based on the assumption that the optics are entirely linear outside of the insert region, and therefore the error-driven dynamics will not accrue during periods of linear transport. High order effects in the linear focusing section (i.e., nonlinear space charge or nonlinear field components) are omitted here. This section summarizes the cases studied; a more complete description of the approach is found in [24].

First, gross misalignments of the channel were considered. This is the case in which the channel has a tilt with respect to the closed orbit. The amplitude of distortion is

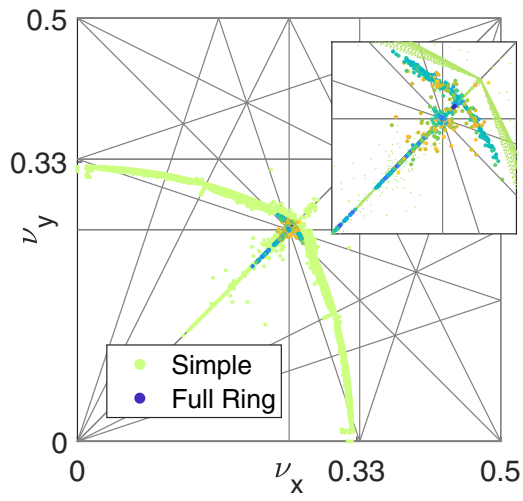

(a) Tune footprint with up to fourth order resonance lines indicated. For color axis refer to scale in plot below.

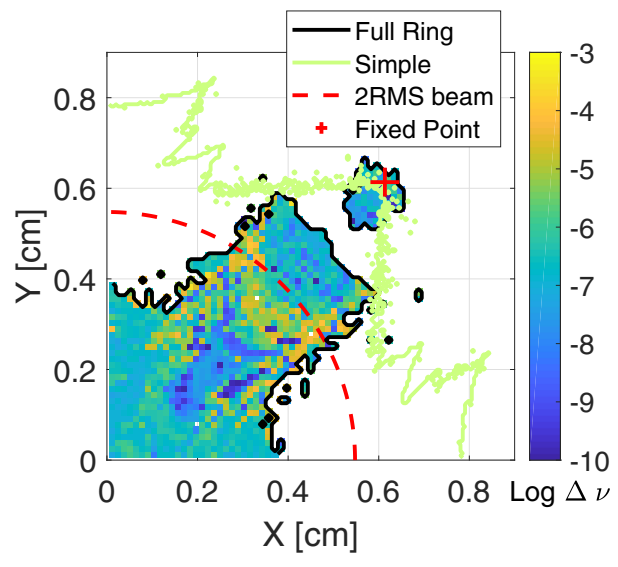

(b) Configuration space plot of aperture and resonant structure.

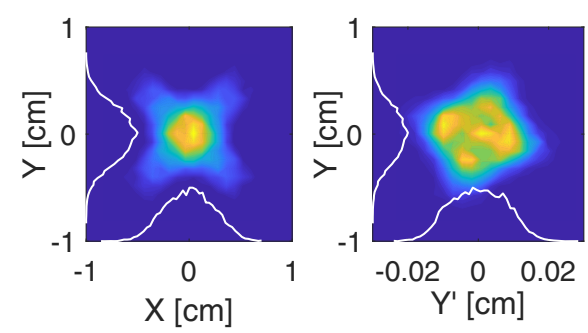

(c) Snapshot of beam distribution at beam waist. Color axis scales linearly with beam density.

FIG. 8. WARP PIC calculation for octupole lattice with peak field $50 \mathrm{~T} / \mathrm{m}^{3}\left(\kappa=3984 \mathrm{~m}^{-1}\right)$ and fractional tune 0.26 after 1024 turns. In (b) and (a), results are compared to the equivalent simple model results. 


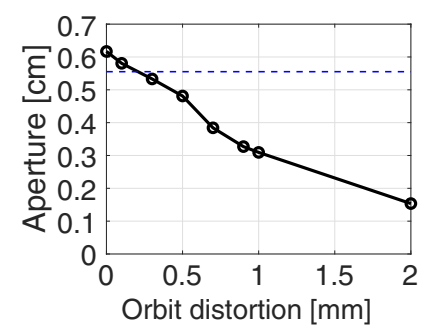

(a) Radial aperture vs. orbit error. Dashed line is $80 \%$ aperture.

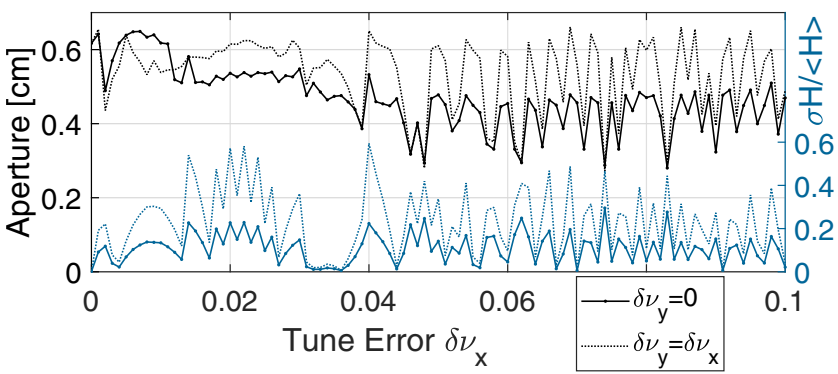

(c) Radial aperture and invariant conservation vs. tune error. Invariant is calculated for a subset of low-amplitude stable particles.

FIG. 9. Effect of steering/focusing errors on octupole lattice stability in simple model with $\beta_{*}=0.3 \mathrm{~m}$, peak octupole strength $50 \mathrm{~T} / \mathrm{m}^{3}$. Aperture limit is defined by largest circular area that contains only stable particles.

parametrized as the transverse offset $\Delta x$ of the closed orbit at insert entrance. Only cases with zero average distortion, $<\Delta x(s)>=0$. The total dynamic aperture decreases dramatically with increasing closed orbit distortion, shown in Fig. 9(a), as particles are placed beyond the boundary of stability. Orbit distortions $<0.2 \mathrm{~mm}$ are required to preserve $80 \%$ dynamic aperture by area. The best orbit control measured over a single $20^{\circ}$ UMER section is reported in Table IV. Clearly, the measured distortion far exceeds the desired tolerance, particularly in the horizontal plane.

The main culprit is not mechanical errors, which were corrected and surveyed prior to these measurements, but rather an effective misalignment due to ambient magnetic fields, which provides $\sim 20 \%$ of the total horizontal bending in the ring. As shown in Fig. 9(b), adding a constant bending field to the simple orbit-error model shows a weak dependence up to $400 \mathrm{mG}$, which is the average vertical background field in the ring. For $80 \%$ of the dynamic

TABLE IV. Measured centroid position (in millimeters) at proposed octupole insert location on first turn, measured from center of $20^{\circ}$ section using first-turn quadrupole response data.

\begin{tabular}{lrccc}
\hline \hline Axis & \multicolumn{1}{c}{$-24 \mathrm{~cm}$} & $-8 \mathrm{~cm}$ & $8 \mathrm{~cm}$ & $24 \mathrm{~cm}$ \\
\hline $\mathrm{X}$ & $-0.18 \pm 0.16$ & $0.69 \pm 1.11$ & $-0.14 \pm 0.49$ & $-0.56 \pm 0.41$ \\
$\mathrm{Y}$ & $0.10 \pm 0.03$ & $0.05 \pm 0.99$ & $-0.08 \pm 0.54$ & $-0.06 \pm 0.88$ \\
\hline \hline
\end{tabular}

aperture to be preserved, background fields $<100 \mathrm{mG}$ (in one plane) are desired.

The simple model was also used to interrogate dependence on focusing errors in the linear-focusing lattice. Assuming single-particle dynamics dominate in the linear sections, a phase shift $\sigma_{x}=n \pi+\delta \sigma_{x}$ was added to the thin kick transfer matrix between insertions:

$T_{x}=\left[\begin{array}{cc}\cos \sigma_{x}-\alpha_{x} \sin \sigma_{x} & \beta_{x} \sin \sigma_{x} \\ -\frac{1-\alpha_{x}^{2}}{\beta_{x}} \sin \sigma_{x}-\frac{2 \alpha_{x}}{\beta_{x}} \cos \sigma_{x} & \cos \sigma_{x}-\alpha_{x} \sin \sigma_{x}\end{array}\right]$

where $\delta \sigma_{x}=0$ for the ideal case, and $\alpha_{x}, \beta_{x}$ are the Courant-Snyder parameters. The phase error in the $y$-plane is applied in the same way.

The effect of tune errors on dynamic aperture has a weaker dependence than the centroid errors, as shown in Fig. 9(c). In general, lattice tunes within $<0.02$ of the optimal value preserved $80 \%$ of the stable aperture (by radius), and errors out to 0.1 resulted in, on average, $30 \%$ loss but without a strong dependence on error magnitude. On the other hand, conservation of the invariant quantity depends more strongly, with variations $>20 \%$ for singleplane tune errors $>0.01$. In general, it was found that a "symmetric error", in which $\nu_{x}-\nu_{y}$ is small, had less effect than errors with asymmetric tunes. This is consistent with results discussed in Ref. [4], in which equal chromatic errors are noted to preserve the required symmetry in the invariant quantities.

Finally, replacing the "ideal" octupole insert [pure octupole field with perfect $\beta^{-3}(s)$ dependence] with the field "as designed" in Sec. III A (generated by Biot-Savart integration) showed a very marginal decrease in stable aperture $(<1 \%)$ and a mild increase in the fluctuation of $H_{N}(+1 \%)$.

In brief, analysis of a simple octupole lattice shows strong sensitivity to orbit distortion that exceeds the current threshold of demonstrated orbit control in UMER. This motivates the installation of additional steering compensation coils to cancel the ambient fields. The sensitivity to lattice tune errors is less crucial (to the levels investigated). Although the operating point should be tuned as close to the QI condition as possible, the relatively relaxed tolerance is promising for extension to space-charge dominated beams with intrinsic tune spread.

\section{DISCUSSION AND CONCLUSION}

This paper presents an overview of the progress and prospects for testing the feasibility of nonlinear optics at UMER. Section III describes a configuration that supports invariant-conserving orbits at tune spreads up to $\Delta \nu=$ 0.13 (rms 0.015) with a single octupole insert. The primary limitation of the design as presented is the strength of the predicted effect, which is much lower 
than the fully-integrable system (up to unity tune spread with multiple insertions [8]). This is partially due to the high-density, many-bend lattice that required a perhaps shorter-than-optimal nonlinear insert. For applications beyond proof-of-principle, more insertions are desired. The results presented here regarding tolerances and expected tune spreads are consistent with similar studies done for the octupole experiment at IOTA [16].

Some of the technical challenges confronted during this design process are specific to UMER. These include the large orbit distortion and unshielded ambient magnetic field at the time of this work. Simulation-based predictions of sensitivity has motivated the installation of ambient-fieldcancelling corrector coils (that are near completion at the time of this submission). Finally, although many quadrupoles are available for optimizing linear focusing, the lack of dispersion matching at injection limits control of dispersion in the octupole section. In general low energy spread is expected for both coasting-beam and rf-confined experiments $(\delta p \sim 0.001)$, but the momentum acceptance should be examined in both simulation and experiment.

Sensitivity to resonance near the fourth order-stopband limits the maximum tune spread that can be achieved in the UMER octupole lattice. A pertinent question for design of strong octupole lattices is whether this sensitivity is mitigated when the invariant-conserving condition is met. Although observed in full-ring PIC simulations, this resonance led to no loss in the simple model, where the ratio of nonlinear to linear focusing is much larger. While it is unsurprising that the octupole resonance is strongly driven, it may be possible for the tune footprint to span fourth order stop-bands if decoherence time balances the resonant growth rate. Finally, the need to operate with particles near the dynamic aperture limit, which corresponds with highly tune-shifted orbits, is in contrast to typical accelerator operations, in which dynamic aperture is maximized. This motivates the need for tightly controlled closed orbit in the insertion region.

Encouragingly, some design aspects were much easier to reconcile than initially expected. For a high emittance beam we require modest octupole strength well below the upper limits (determined by threshold for heat-damage to the PCB circuits). Also, the purity and precision of the octupole channel does not appear to have a large effect on the dynamics; a model with Biot-Savart generated fields showed only mild degradation of invariant conservation and stable aperture. Finally, the system has weak dependence on errors in lattice phase advance, which is promising with respect to internal tune spread due to space charge. Continuing to advance understanding of the dependence of invariants and stability on space charge will be important in determining the future of integrable optics as a method to improve transport of high intensity beams.

The design presented here will be the starting point for initial experimental runs. Significant flexibility has been preserved in the linear-focusing optics, which will accommodate operating points with higher fractional tunes as well as multi-insert experiments. Observing stable beam transport at the design operating point, in tandem with extending the simulation studies to include additional errors and driving terms, will continue to build intuition for design and operation of integrable optics machines. Ultimately, the goal of the UMER nonlinear optics program is to extend into denser space charge regimes (i.e., row 2 in Table II), following demonstration at the $\mu \mathrm{A}$ level. Although this paper only considered transport in the lowest-charge case, the apparatus as designed supports examination of the transition from the single-particle regime described by the NLIO theory to that in which space charge becomes a significant factor.

\section{ACKNOWLEDGMENTS}

Thanks to Santiago Bernal for developing low-current beam capability at UMER, as well as a very careful proofreading of this manuscript. Also many thanks to Levon Dovlatyan, Rami Kishek, Dave Sutter, Eric Montgomery for helpful discussions and technical expertise. This project has benefited greatly from communication with the IOTA program at FNAL. Funding for the work was provided through DOE-HEP (Award No. DESC0010301), NSF (Grant No. PHY1414681) and the NSF GRFP (Grant No. DGE1322106). This manuscript has been authored by UT-Battelle, LLC, under Contract No. DEAC0500OR22725 with the U.S. Department of Energy.

[1] A. W. Chao, Physics of Collective Beam Instabilities in High Energy Accelerators (John Wiley \& Sons, New York, 1993).

[2] S. D. Webb et al., in Proceedings of the 4th International Particle Accelerator Conference, IPAC-2013, Shanghai, China, 2013 (JACoW, Shanghai, China, 2013).

[3] V. Danilov and S. Nagaitsev, Nonlinear accelerator lattices with one and two analytic invariants, Phys. Rev. ST Accel. Beams 13, 084002 (2010).

[4] S. Webb, D. Bruhwiler, A. Valishev, S. Nagaitsev, and V. Danilov, in Proceedings of the ICFA Advanced Beam Dynamics Workshop on High-Intensity and High-Brightness Hadron Beams (JACoW, East-Lansing, MI, 2014).

[5] S. Nagaitsev and A. Valishev, in Proceedings of the 3rd International Particle Accelerator Conference, New Orleans, LA, 2012 (IEEE, Piscataway, NJ, 2012).

[6] C. Hall, D. Bruhwiler, N. Cook, R. Kishek, and S. Webb, Impact of space charge on beam dynamics and integrability in the IOTA ring, Proceedings of the Space Charge Workshop in Darmstadt, Germany (2017), https://indico .gsi.de/event/5600/session/5/contribution/19.

[7] J. Eldred and A. Valishev, in Proceedings of the Int'l Particle Accelerator Conference (JACoW, Copenhagen, Denmark, 2017). 
[8] S. Antipov et al., IOTA (Integrable Optics Test Accelerator): facility and experimental beam physics program, J. Instrum. 12, T03002 (2017).

[9] R. A. Kishek et al., The university of maryland electron ring program, Nucl. Instrum. Methods Phys. Res., Sect. A 733, 233 (2014).

[10] S. Bernal et al., in Proceedings of the Advanced Accelerator Concepts Workshop (AIP, National Harbor, MD, 2017), Vol. 1812, p. 110006.

[11] J. Wei et al., Low-loss design for the high-intensity accumulator ring of the Spallation Neutron Source, Phys. Rev. ST Accel. Beams 3, 080101 (2000).

[12] K. Hamilton, L. Dovlatyan, D. Matthew, D. Sutter, S. Bernal, T. A. Antonsen, and B. Beaudoin, in Proceedings of the Advanced Accelerator Concepts Workshop (IEEE, Breckenridge, Colorado, 2018).

[13] B. Beaudoin, I. Haber, R. Kishek, S. Bernal, and T. Koeth, Long path-length experimental studies of longitudinal phenomena in intense beams, Phys. Plasmas 23, 056701 (2016).

[14] W. Zhang, S. Bernal, H. Li, T. Godlove, R. Kishek, P. O'Shea, M. Reiser, and V. Yun, Design and field measurements of printed-circuit quadrupoles and dipoles, Phys. Rev. ST Accel. Beams 3, 122401 (2000).

[15] F. O'Shea, R. Agustsson, Y. Chen, E. Spranza, D. Martin, and J. Mcnevin, in Proceedings of the Int'l Particle Accelerator Conference (JACoW, 2015), pp. 724-727.

[16] S. Antipov, S. Nagaitsev, and A. Valishev, Single-particle dynamics in a nonlinear accelerator lattice: attaining a large tune spread with octupoles in IOTA, J. Instrum. 12, P04008 (2017).

[17] R. Conte, M. Musette, and C. Verhoeven, Explicit integration of the Hénon-Heiles Hamiltonians, J. Nonlinear Math. Phys. 12, 212 (2005).

[18] H. Baumgartner, S. Bernal, I. Haber, T. Koeth, D. Matthew, K. Ruisard, M. Teperman, and B. Beaudoin, in Proceedings of the North American Particle Accelerator Conference (JACoW, Chicago, IL, 2016), pp. 503-506.

[19] H. Baumgartner, K. Ruisard, D. Matthew, T. Koeth, I. Haber, and B. Beaudoin, in Proceedings of the Int'l Particle Accelerator Conference (JACoW, Vancouver, Canada, 2018).

[20] S. Webb, D. Bruhwiler, S. Nagaitsev, V. Danilov, A. Valishev, D. Abell, A. Shishlo, K. Danilov, and J. Cary, Effects of nonlinear decoherence on halo formation (unpublished).

[21] B. L. Beaudoin, S. Bernal, C. Blanco, I. Haber, R. A. Kishek, T. Koeth, and Y. Mo, Modeling HIF relevant longitudinal dynamics in UMER, Nucl. Instrum. Methods Phys. Res., Sect. A 733, 178 (2014).

[22] Y. Cui, Ph.D. thesis, University of Maryland, 2004.

[23] S. Bernal, B. Beaudoin, L. Dovlatyan, S. Ehrenstein, I. Haber, R. A. Kishek, E. Montgomery, and D. Sutter, Low space-charge intensity beams in UMER via collimation and solenoid focusing, arXiv:1810.04264v1.

[24] K. Ruisard, Ph.D. thesis, University of Maryland, College Park, MD, 2018.

[25] A. Friedman, R. Cohen, D. Grote, S. Lund, W. Sharp, J. Vay, I. Haber, and R. Kishek, Computational methods in the warp code framework for kinetic simulations of particle beams and plasmas, IEEE Trans. Plasma Sci. 42, 1321 (2014).

[26] K. J. Ruisard, H. Baumgartner, B. Beaudoin, S. Bernal, B. Cannon, L. Dovlatyan, I. Haber, and T. Koeth, in Proceedings of the Int'l Particle Accelerator Conference (JACoW, Vancouver, Canada, 2018), pp. 3585-3588.

[27] H. Li, computer code MAGLI, 2005.

[28] J. Laskar, in Proceedings of the 2003 Particle Accelerator Conference, Portland, OR (IEEE, New York, 2003), pp. 378-382.

[29] K. Ruisard, B. Beaudoin, I. Haber, D. Matthew, and T. Koeth, in Proceedings of the Int'l Computational Accelerator Physics Conference (JACoW, Key West, FL, 2018). 